Anaesthesist 2022 $\cdot$ 71:168

https://doi.org/10.1007/s00101-021-01071-x

Online publiziert: 7. Dezember 2021

(c) Springer Medizin Verlag $\mathrm{GmbH}$, ein Teil von

Springer Nature 2021
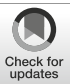

\section{Retraction Note: Volumenersatz beim schwerkranken Intensivpatienten}

\section{Keine klassische Übersicht}

J. Boldt

Klinik für Anästhesiologie und Operative Intensivmedizin, Klinikum der Stadt Ludwigshafen, Ludwigshafen, Deutschland
Die Online-Version des Originalartikels ist unter https://doi.org/10.1007/s001010050625 zufinden.

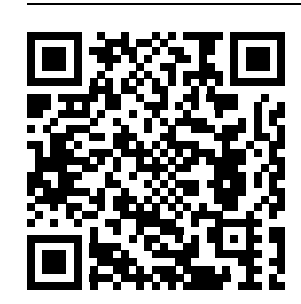

QR-Code scannen \& Beitrag online lesen

\section{Retraction Note to: \\ Anaesthesist 1998}

https://doi.org/10.1007/s001010050625

Die Schriftleitung von Der Anaesthesist hat diesen Artikel zurückgezogen, weil seit seiner Veröffentlichung 5 der Artikel [1-5], auf denen diese Übersicht basiert, zurückgezogen wurden. Diese Artikel werden in der Übersicht als Referenzen 5, 6, 7, 8 und 11 zitiert. Der Autor hat auf die Kontaktaufnahme des Verlags über diese Rücknahme nicht geantwortet.

\section{Korrespondenzadresse}

\section{Prof. Dr. J. Boldt}

Klinik für Anästhesiologie und Operative Intensivmedizin, Klinikum der Stadt Ludwigshafen

Bremserstraße 79, 67063 Ludwigshafen, Deutschland

\section{Literatur}

1. Boldt J, Zickmann B, Ballesteros BMet al (1992) Einfluss von fünf verschiedenen Priming-Lösungen auf die Thrombozytenfunktion bei Patienten, die sich einer Herzoperation unterziehen. Anästhesie Analg 74(2):219-225

2. Boldt J, Knothe C, Zickmann B et al (1993) Einfluss verschiedener intravaskulärer Volumentherapien auf die Thrombozytenfunktion bei Patienten mit kardiopulmonalem Bypass. Anästhesie Analg 76(6):1185-1190

3. Boldt J, Heesen M, Welters I et al (1995) Beeinflusst die Art der Volumentherapie die endothelial bedingte Gerinnung bei kritisch Kranken? Br J Anaesth 75(6):740-746

4. Boldt J, Zickmann B, Rapin J et al (1994) Einfluss des Volumenersatzes mit verschiedenen HESLösungen auf den mikrozirkulatorischen Blutfluss in der Herzchirurgie. Acta Anaesthesiol Scand 38(5):432-438

5. Boldt J, Müller M, Mentges D et al (1998) Volumentherapie bei Schwerstkranken: Gibt es einen Unterschied? Intensive Care Med 24:28-36 